\title{
Uncovering motivators and stumbling blocks: Exploring the clinical research experiences of speech-language pathologists
}

EMMA FINCH ${ }^{1,2,3}$, PETREA CORNWELL ${ }^{4,5}$, EMILY NALDER $^{6,7}$, ELIZABETH WARD $^{2,3}$

${ }^{1}$ Speech Pathology Department, Princess Alexandra Hospital, Woolloongabba, Queensland, Australia

${ }^{2}$ Division of Speech Pathology, School of Health and Rehabilitation Sciences, The University of Queensland, Queensland, Australia

${ }^{3}$ Centre for Functioning and Health Research, Metro South Hospital and Health Service, Queensland, Australia

${ }^{4}$ Allied Health Research Collaborative, Metro North Hospital and Health Service, Chermside, Queensland, Australia

${ }^{5}$ Behavioural Basis of Health, Griffith Health Institute, Griffith University, Mt Gravatt, Queensland, Australia

${ }^{6}$ School of Health and Rehabilitation Sciences, The University of Queensland, Queensland, Australia

${ }^{7}$ Rotman Research Institute, Baycrest, Toronto, Canada

Key words: speech-language pathology; qualitative content analysis; focus group; clinical research; research engagement

Running head: SLP clinical research experiences

Corresponding author:

Emma Finch, Speech Pathology Department, Princess Alexandra Hospital

Ipswich Road Woolloongabba QLD 4102, Australia, Telephone: +61 73896 3133, Email:

e.finch@uq.edu.au 


\begin{abstract}
Healthcare providers increasingly expect that allied health staff will not only translate research evidence into their clinical practice, but also generate research. Little is known about how well prepared clinicians are to meet these expectations. Research suggests that allied health professionals, including speech-language pathologists, have moderate levels of interest in research, but only little to moderate experience participating in research activities. The present study aimed to explore the experiences and attitudes of speech-language pathologists in regards to undertaking research in their clinical settings. Focus groups were conducted with 21 practising speech-language pathologists (13 research engaged, 8 not research engaged). The focus groups were transcribed and the data analysed qualitatively using content analysis. Two overarching themes mediated research engagement. Engagement in research was shaped by whether participants overcame any "fear" of research, and the unique characteristics of their clinical context. Contextualising and further shaping participants' experiences of these themes were personal factors, such as initiative and proactivity. The success of increasing the research engagement of the speech-language pathology workforce may be contingent on providing clinicians with more exposure to research opportunities and mentors as well as ensuring organisational structures are in place to encourage, support and facilitate research.
\end{abstract}




\section{Introduction}

In the past decade, there has been increased recognition of the value of research to the healthcare system, with the translation of research into clinical practice identified as a key priority not only by governments worldwide, but also by independent international bodies such as the World Health Organization (WHO) (Australian Government, 2013; Canadian Institutes of Health Research, 2004; NHMRC, 2013; Peters, Tran, \& Adam, 2013; Straus, Tetroe, \& Graham, 2009). Despite this global attention, it is acknowledged internationally that there remains a substantial divide between the generation of health research and the actual provision of healthcare services (Kitson, Powell, Hoon, Newbury, Wilson, \& Beilby, 2013; NHMRC, 2013; Straus et al., 2009). In Australia, this translation gap continues to exist despite a high level of interest in evidence based practice (EBP) and research activities (Queensland Government, 2007, 2013).

In order to foster change, numerous national allied health professional groups (Australian Physiotherapy Association, n.d.a.; Occupational Therapy Australia, 2013; Speech Pathology Australia, 2012) and healthcare settings in Australia (Queensland Government, 2007, 2013; Queensland Industrial Relations Commission, 2007) now actively promote and support high levels of research engagement by their members. Speech Pathology Australia, the peak professional body of speech-language pathologists (SLPs) in Australia, specifically built engagement in research activities into a number of professional standards within its Competency-Based Occupational Standards (CBOS) document, including requiring SLPs to have an awareness of current research and participate in research activities (Speech Pathology Australia, 2011). Despite this high level of research promotion, there is little evidence to guide how best to achieve such increased research integration into clinical services, or what factors motivate clinicians to embrace a research culture within their clinical settings.

Lessening the research translation gap and ensuring the research findings are incorporated into everyday clinical practice is a vital step towards developing more effective healthcare systems and ensuring that patients receive the best possible care, potentially leading to improved health 
outcomes throughout the world (Canadian Institutes of Health Research, 2004). One approach to lessen the research generation/implementation divide and to facilitate the translation of new research evidence into routine clinical practice is to develop a healthcare workforce that generates research (Australian Government, 2013). In Australia, some healthcare organisations have endeavoured to foster a culture of research generation through dedicated research positions and establishing partnership arrangements with major local universities (Queensland Government, 2012). However, the readiness of the clinical workforce to actively participate in research alongside these initiatives is dependent on two factors: (1) organisational structure (O'Connor \& Pettigrew, 2009) (including time and funding constraints), and (2) interest and a skill set distinct from that required in clinical practice (Finch, Cornwell, Ward, \& McPhail, 2013; Reid, Farmer, \& Weston, 2006; Reid, Farmer, \& Weston, 2007). EBP research has revealed that the workplace environment has been documented as a barrier to EBP implementation (O'Connor \& Pettigrew, 2009), particularly with respect to a lack of time for research implementation (Jette et al., 2003; Nail-Chiwetalu \& Bernstein Ratner, 2007; O'Connor \& Pettigrew, 2009).

Previous survey research of allied health professionals (including SLPs) (Finch et al., 2013; Queensland Government, 2013; Stephens, Taylor, \& Leggat, 2008) and primary healthcare workers (Reid et al., 2006) working in clinical, non-research roles has focused on possession of research skills and interest. Without exception, these studies document that interest in conducting research is higher than respondents' self-rated research experience. Furthermore, the highest level of clinician confidence is reported in activities that align most closely with clinical duties, such as finding relevant literature (Finch et al., 2013; Queensland Government, 2013; Reid et al., 2006; Stephens et al., 2008).

Research has revealed that SLPs are one allied health professional group highly interested in research. In their study of 132 allied health practitioners, Stephens et al (2008) reported that the small group of SLPs $(n=15)$ involved in the study reported higher levels of interest in undertaking clinical research activities than other allied health groups in the cohort (including social workers, physiotherapists, and dieticians). Similarly, a recent large cohort survey of SLPs $(n=137)$ confirmed 
that overall, SLPs indicated strong interested in participating in research (Finch et al., 2013). Despite this interest, Finch et al (2013) found that the majority of respondents reported that they possessed limited experience and low levels of confidence with actually conducting research, suggesting that they felt they did not have the skills required to undertake research. Furthermore, these respondents also reported limited engagement in research activities beyond finding and reviewing literature (Finch et al., 2013). The study found only a small sub-set of participants who were highly interested and highly engaged in research activities (Finch et al., 2013).

Reducing the mismatch between interest and skill level is imperative to gaining increased clinician engagement in research; however, further research is needed to explore the nature of this mismatch by elucidating factors that motivate and/or hinder research engagement. Qualitative methods, in particular focus groups, provide an ideal avenue for this purpose. The interactive and dynamic nature of focus groups may enable participants to explore their attitudes comprehensively, with participants' comments during the focus group serving as a stimulus to prompt other participants (Coenen, Stamm, Stucki, \& Cieza, 2012). The aim of the present study, therefore, was to explore the experiences and attitudes of SLPs in regards to undertaking research in their clinical settings.

Methods

Ethical approval was obtained from The University of Queensland Human Research Ethics Committee.

\section{Participants}

Practising SLPs employed by the state-based public healthcare provider in Queensland, Australia were eligible to participate in the study. To explore the study research question purposeful representative sampling (Marshall, 1996) was employed to identify SLPs engaged or not engaged in research activities, enabling a variety of experiences and perceptions towards conducting clinical 
research to be examined. Potential participants were recruited through an email invitation disseminated via a SLP leadership network. Members of the network forwarded the email invitation to colleagues who then made contact with the research team. A series of focus groups were conducted until saturation of the data occurred (Coenen et al., 2012). Saturation of the data was deemed to have occurred when no new information emerged from subsequent groups.

Twenty-one SLPs participated in a series of 6 focus groups; 13 participants were currently undertaking research activities (classified as "engaged in research"), while 8 participants had not previously and were not currently undertaking formal research activities (classified as "not engaged in research"; See Table 1 for demographic information). Participants who were classified as "engaged in research" reporting varying levels of participation in research including leading their own research projects, participating in multidisciplinary projects with a team of colleagues, collecting data for projects led by other people, and clinical evaluations. All participants were female and predominantly employed in a permanent full-time basis with entry-level qualifications (bachelor, bachelor with honours, and graduate entry masters). Participants were from both metropolitan ( $\mathrm{n}=14)$ and nonmetropolitan $(n=7)$ regions, and from a range of workplace settings; however, hospitals with an adult caseload were the predominant work environment. Participants reported a continuum of clinical experience spanning from less than 5 years to one participant reporting more than 30 years of experience.

\section{[Insert Table 1 near here]}

\section{Data collection}

Each focus group was conducted by a member of the research team with a second individual present to provide assistance and document key points. The focus groups were conducted via video or teleconferencing (with teleconferencing referring to an audio only connection) due to participants' diverse geographical locations and clinical constraints. These recordings were stored for subsequent transcription and analysis. Locally employed participants or those without access to video or teleconferencing facilities were provided with the option of being present at the base site. The 
majority of groups consisted of a mixture of remote and face-to-face participants. To avoid any potential issues in terms of turn-taking imbalances if some participants were physically present in a group and others were remote, the researchers took care to ask each participant's viewpoint if they had not expressed a view during each question before moving on to the next question or discussion area. No participation imbalances were noted by the researchers across the different attendance modes. The focus groups were divided into two types: one series of groups consisted of participants currently engaged in research in their clinical setting, and the second series of groups consisted of participants who were not engaged in research. This division of groups was adopted to enable both perspectives (that is currently undertaking research and not undertaking research) to be explored in detail without one perspective overwhelming the other. Six focus groups were conducted in total (three with participants currently engaged in research activities, three with participants not engaged in research activities). Each focus group consisted of 2 - 5 participants and was on average 46 minutes (range: $14-64$ minutes). All focus groups commenced with an open-ended question (as per Krueger \& Casey, 2000). For the group currently doing research, this was “Tell us about your experiences doing research in a clinical setting?", while for the group not currently doing research this was "You have indicated that you have not done research to date. Could you tell us more about this?" These questions were designed to stimulate a discussion about participants' experiences and attitudes towards conducting clinically-based research. As each focus group progressed, a number of questions were asked to cover topics such as motivators or facilitators to undertaking research, benefits of undertaking research, challenges you see to undertaking research, suggestions for overcoming or minimising these challenges, and suggestions to encourage more clinicians to undertake research. The questions served as a topic guide and were consistent across the groups.

Data analysis

All focus groups were transcribed verbatim. Qualitative content analysis (Graneheim \& Lundham, 2003) was then used to analyse the transcripts. To increase familiarity with the data, each 
transcript was first read through completely, noting (in an audit trail) overall impressions of the data and any concepts which were more dominant in each transcript. The focus group transcripts formed the unit of analysis and were then examined individually highlighting condensed meaning units, which are defined as participants' statements pertaining to the same underlying content or meaning. Meaning units were labelled with a code to describe the content and thus several meaning units comprised one code. Both manifest content (the explicit components of the text) and latent content (implied meaning in text) were coded. To illustrate these concepts two meaning units coded as lack of time are presented. The meaning unit, "finding the time to do it" reflects manifest content and "[research] always gets put to the backburner in terms of prioritisation and client time always came first" captures the latent idea that there is insufficient time for research. Both of these meaning units ultimately contributed to the theme "clinical context shapes research".

The initial coding was conducted by one member of the research team (EN). Coded transcripts were then checked by a second researcher $(\mathrm{EF})$. As there was a high level of agreement in coding only minor revisions to the coding framework were required based on this peer checking. For example, renaming codes to reflect consensus codes formed during discussion between the two researchers. Transcripts were then re-read to ensure the codes accurately reflected the content of the meaning unit. Any meaning units not initially coded were also classified at this time. Once all transcripts had been coded and checked the data was extracted (i.e., data was copied from the transcript and placed into a separate document containing all statements related to that code). This allowed for all coded statements to be examined together. In this way the various codes were compared and classified into themes and sub-themes. For example codes such as "lack of research skills" and "research training" were grouped under a broader theme of "breaking down the fear of research". A descriptive summary of the themes was developed and discussed amongst the research team which lead to further refinements of the themes and their definitions. For example, it was through discussions amongst the team that the theme of "personal factors" was identified as a latent idea shaping the clinicians' experiences of doing research. Once the themes had been finalised the descriptive summary was reviewed by members of the research team in the context of the audit trail 
thereby comparing initial impressions recorded during data analysis with resulting themes. No changes to the themes were made at this stage as the research team agreed they captured the overall essence of the transcripts. Microsoft @ ${ }^{\circledR}$ word was used for documenting all components of the analysis.

Rigour

To ensure that rigour was upheld during data analysis the research team met regularly to review and discuss the transcripts and emerging themes. This peer checking process occurred at all stages of the data analysis process, including identification of meaning units, coding, and classification and refinement of themes and sub-themes. Any discrepancies or disagreements during the peer checking process were discussed amongst the research team until consensus was reached. An audit trail relating to decisions made relating to the study methodology, analysis and underlying theoretical framework was maintained (Koch, 2006).

Results

Two overarching themes emerged from the transcripts: breaking down the fear and clinical context shaping research, representing a continuum of positive and negative experiences. Each theme contained a number of sub-themes (For Breaking down the fear this involved: experiential research training, availability of support and training, and past experiences shaping confidence level; For Clinical context shaping research this involved: organisational support for research, and priorities for research in clinical role). Further contextualising the participants' contribution to the two overarching themes was the influence of personal factors. For each supporting quote, participant identification codes are identified in brackets, with EP representing "Engaged in research participant" and NP representing "Not engaged in research participant."

\section{Overarching theme 1: Breaking down the fear}


Breaking down the fear referred to the idea that there was low confidence amongst participants about their ability to successfully undertake research tasks and that overcoming this low confidence and apprehension was a crucial element of engaging in research. The ability to overcome the fear of research served as an invisible barrier separating those participants who had engaged in research from participants who had not participated in research activities. One participant alluded to this by commenting:

"I'm still a novice researcher even though lots of other people go - oh well you are doing research you know what you are doing - but I don't think I actually do but I'm just willing to step out into the dark and admit that I don't know what I am doing I think that is the difference." [EP10]

The key elements that helped to break down the fear of research and facilitate research engagement were experiential research training, availability of support and training, and past experiences shaping confidence level.

\section{Sub-theme 1: Experiential research training}

Participants currently engaged in research activities typically reported having received either formal (e.g., workshops) or informal (e.g., undertaking research activities with a more research experienced colleague) research training. Experiential research training, that is the acquisition of research skills obtained during hands on participation in research tasks under the guidance of a more experienced researcher, was highly valued. To break down their fear of research, participants desired research training with a more experienced researcher who could gradually step them through the research process. A component of the fear towards research was the perception by some participants that they lacked the pre-requisite research skills that more experienced researchers possessed. One participant commented:

"I'm doing a group research [project] with a researcher who has experience and who is sharing that experience with us and using it as sort of a teaching tool and I think that has been really beneficial.” [EP12] 
Conversely, a lack of research training was viewed as a key obstacle preventing participants who were not engaged in research from partaking in research-related activities: "You are not ever going to go and do your own research project off the bat immediately." [NP05] This highlighted the idea that participants desired gradual support through practical experience and mentoring to build research skills.

Successful experiential research training that equipped participants with sufficient skills and support to break down the fear of research encompassed a range of activities. Participating in research to varying levels, ranging from recruiting for someone else's study to writing ethics applications and submitting grant applications, provided foundational research training for many participants engaged in research. Participants engaged in research also commented about the importance of a research mentor or support person to serve as a role model, provide practical experience and/or facilitate research: "Just being able to share and being supported by the right people." [EP11] In contrast, the absence of a research mentor or support person featured prominently in the transcripts of participants who were not engaged in research activities: "I would be interested in if you did have someone with that experience, in doing more research you know whether they'd be a mentor or a joint sort of partner in that research I think that would certainly be helpful.” [NP02]

Several participants engaged in research identified collaborating with colleagues or external organisations as a method of sharing the workload and skill set. Some participants currently engaged in research reported actively seeking out collaborations to reduce the individual workload, while simultaneously maximising the available expertise: "I really got a lot out of it... because I was doing it with this other colleague who I get along with really well, we shared the workload and we did a lot of it together so that we felt like two people who didn't have a clue was better than one person who didn't have a clue." [EP10]

Many participants, regardless of their level of research engagement, raised the idea that training in specific research skills at an appropriate time was essential to facilitate and support clinically-based research activities. These specific skills included formulating a research question, grant writing, ethics and statistics. Participants acknowledged that while they received some research training during their university studies, this knowledge was not retained over time: 
"It is a bit frustrating because you do know hey I did learn this but when you're learning it without any context you are very young, you are learning so many other things you don't really take it in. It is not until you have the living breathing context and have an idea of what you want to achieve with the research that all that stuff becomes relevant and by then you have forgotten it all." [EP05] This again highlighted the importance that participants placed on experiential research training at a time when they desired the specific research skills.

\section{Sub-theme 2: Availability of support and training}

To overcome the fear of research, participants required support; however, the availability of support and training varied based on contextual and personal factors. Some participants reported that research in their workplace was enabled and encouraged by a facilitator or in some cases a conjoint researcher linked to a university: "In my workplace we actually have a conjoint researcher with the University and that allows us, I think, to probably do research more easily." [EP09] In contrast, some participants not engaged in research reported a sense of feeling disconnected: "It's more challenging in the community because you aren't based with the speech-language pathologists that are at the hospital so you don't know what is going on" [NP03], and were unaware about research projects occurring in their workplace: "I don't know what is current in my area and that sort of information doesn't seem to be available." [NP03] When confronted with a similar challenge, many participants engaged in research reported proactively attempting to seek out further support and knowledge through external organisations, particularly for assistance with statistical analysis or the write up of results. "And the big problem I find with the research projects is getting the statistical support which I have done through linking with Universities or particularly particular colleagues." [EP08] These differing responses to the similar challenges in undertaking research highlight that personal factors (e.g., initiative and motivation) may play an important part in overcoming barriers to obtaining research support and skills. 


\section{Sub-theme 3: Past experiences shaping confidence level}

Past experiences conducting research played a pivotal role in determining whether participants experienced fear of research by shaping confidence levels. Participants who reported active involvement with clinical research talked of favourable experiences that prompted them to undertake further research activities: "That has been a very positive experience... in the future... I would like to continue research" [EP02], "Very stimulating and rewarding" [EP04]. Positive previous research experiences encompassed formal training (undergraduate or honours degrees), and previous research projects completed in participants' clinical settings. These experiences may have enabled participants to gain not only valuable research skills, but also to successfully complete a project, leading to a sense of confidence with research. Conversely, negative previous research experiences decreased participants' confidence and were viewed as barriers and deterrents to engaging in further research activities. Nonetheless participants engaged in research often reported persevering beyond negative experiences to persist in pursuing their research goals. Two participants commented: "Whenever I have tried to lead research by myself it hasn't gotten very far before it's hit a wall" [EP12]; "Having written a few bids that have been unsuccessful for funding so not being able to get the backfill [I] have had trouble getting things off the ground." [EP13] Despite these negative experiences of solo-research, both of these participants were engaged in research at the time of the focus groups because they had sought out collaborations with more research-experienced colleagues who provided successful, positive research experiences. This reinforces the importance of support to increase confidence and develop skills so that clinicians can conduct future research more independently. The quote also highlights the influence of personal factors, such as proactivity, initiative and motivation, on research engagement, and illustrates the complexity of factors driving engagement in clinical research. While confidence can be affected by negative research experiences, this does not solely dictate subsequent research engagement as a host of other factors, including personal factors and available support and training, also play a determining role.

\section{Overarching theme 2: Clinical context shaping research}


The influence of clinical context on research activities was a second overarching theme that featured prominently in the discussions. This contextual influence stemmed predominantly from the organisational support for research activities and the prioritisation of research in each participant's clinical role.

\section{Sub-theme 1: Organisational support for research}

Participants acknowledged that their organisations greatly influenced their research engagement by presenting both facilitatory and inhibitatory influences: "When you try and do it [research] within a (employing organisation) context it... has its extra opportunities but it also has its extra barriers and challenges as well." [EP10] These challenges originated from a number of sources: "You have got your... local service restrictions and patient restrictions and your time restrictions and that is in an environment that is supported and actually generates a lot of research." [EP08] How participants individually responded to the unique facilitators and challenges presented by their workplace was a key factor determining their level of research engagement. Personal factors, such as proactivity and initiative, played an influential role in this individual response.

Departmental planning and priorities for research varied widely, impacting upon participants' research engagement. Some departments prioritised research highly by identifying research as a departmental goal or by creating dedicated research positions or portfolios. While a few participants reported that they received good levels of support from their line managers and organisations, many reported that a lack of support from higher levels within their organisation constituted one of the dominant barriers to research: "I felt like I couldn't take my clinician hat off to do research." [EP11] Many participants, both engaged and non-engaged in research, reported that initial support for research from their organisation frequently did not translate down to practical support for implementing the research project at a later date: "I think theoretically in the workplace everyone is like yeah research is great, it's fantastic, but practically getting the support for offline time it's not going to happen.” [EP12] 
Information dissemination about research projects occurring within individual departments varied between participants, influencing their research engagement. A lack of knowledge about projects was a barrier to staff engaging in research. "Unless you are actually involved in a project so [or] you are a senior staff member, nobody else was aware of the projects or what was happening so you had no way of contributing or highlighting that you had an interest in what they were doing because you didn't know what was happening." [NP05] This also highlighted the idea that the clinical context shaped the opportunity for experiential research training.

Time was a key factor woven through the discussions. Many participants reported that time constraints from their clinical caseload greatly limited their research engagement:

"Time is a major factor for people, for fulltime staff, trying to do clinical research it is so hard to be able to prioritise time... At the start of the year you write down your ideas and you have this great plan and by May you are back into fulltime swing and it is all just gone." [EP09]

Participants engaged in research reported frequently undertaking research tasks in their own personal time in order to achieve successful completion of their research project, particularly during the writing up stage "You're just scraping by to get all of your data collected within work time and you do a lot of analysis and write-up in your own time and that drags out for ever... it's a weekend and I should be able to have a weekend off." [EP04] Grants provided an opportunity to minimise time constraints by enabling backfilling of their clinical caseloads. However, less clinically experienced participants reported being the backfill for more senior staff to work offline on research tasks, thereby preventing the junior staff members from engaging in research activities themselves. Not surprisingly, participants who had research projects focused around their clinical caseloads encountered time constraints less frequently, particularly during data collection: "It was really easy to do data collection that year because it was part of my routine clinical practice." [EP06] This statement also introduces the idea that research can indeed successfully co-exist with clinical practice. An additional organisational factor that influenced whether participants engaged in research activities was the length 
of time that they had been in their clinical position, with frequent clinical caseload rotations or shortterm temporary clinical positions not considered conducive to research. This barrier appeared to stem from a number of factors including the perceived need by participants to build skills and experience in their clinical caseload before embarking on research activities, clinical time pressures from a new caseload, and in some cases a lack of opportunity from managers to pursue research tasks.

\section{Sub-theme 2: Priorities for research in clinical role}

The clinical context additionally shaped participants' research engagement by influencing the priority of research within each individual speech-language pathologist's clinical role. The majority of participants, regardless of their level of research engagement, viewed research as an important means of improving and validating the services provided to clients: "I do it [research] because it maximises outcomes for my patients and hopefully other patients as well." [EP01] Participants from all levels of research engagement also identified research as fulfilling a need for evidence-based practice (EBP) that could be disseminated and accessed by colleagues: "I am doing something really well during my clinical practice and it works, I feel like I should be able to write about it and share it with others." [NP06] A further factor elevating the priority of research was a personal interest in research along with a desire to acquire new skills and experiences: "In my clinical work where you know I really lost motivation... research offered me you know the capacity to build on my writing skills, to build on analytical skills, to build on my presentation skills" [EP03]; "Being able to do the clinical research is very personally rewarding for me as well as being able to generate answers that aren't there in the literature." [EP04] For some participants, research was also seen as a high priority as it provided them with the opportunity for career progression and new work opportunities, including research higher degrees. Research was also observed as a way of achieving recognition for work as a department and at an individual level.

Despite the majority of participants viewing research as important, the actual prioritisation of research in their daily work was counterbalanced by competing factors related to their clinical context, personal priorities and a fear of research. For some participants, these competing factors were not 
strong enough to curtail research engagement and they actively engaged in research activities, while for other participants these factors posed a perceived insurmountable barrier preventing them from pursuing research activities. An important factor that influenced the relative priority of research activities was that they had to perceive the research as directly relevant to their clinical practice: "A big motivator for me... [is] to research things that are of absolute practical value in the clinical setting in the hospital so that can be sort of ploughed back immediately into clinical practice." [EP13] A number of research-engaged participants were undertaking research projects that they believed were valuable not only to their clinical caseload but also to the wider profession. In contrast, participants were reluctant to engage in research activities that were not seen as "core business" or had limited application to their clinical caseload. Interestingly, the complex nature of many clients and the nature of a clinical context were seen to limit research activities in some environments. In some cases this barrier was related to clinicians who worked with very specific populations experiencing recruitment difficulties due to the time required to identify suitable participants for research projects.

\section{Discussion}

The present study explored the experiences and attitudes of SLPs regarding undertaking research in their clinical settings. While conducted in Queensland, Australia, the results have potential application to healthcare settings throughout the world, given the similar research translation gaps despite healthcare provider and organisational expectations (Australian Government, 2013; Canadian Institutes of Health Research, 2004; Kitson, et al, 2013; NHMRC, 2013; Peters, et al, 2013; Straus et al., 2009). The results of the present study supported previous findings that SLPs value clinically based research and are interested in engaging in research; however, actual participation appeared to be mediated by a fear of research and factors relating to their clinical context. The ability of participants to address the metaphorical wall to participating in research activities and overcoming the challenges were further influenced by personal factors (e.g., initiative, proactivity, interest in research). 
To date, a limited body of literature has addressed the research experiences of SLPs at either a national or an international level, with the majority of this work focusing on EBP. The limited literature available has revealed that many SLPs report low confidence and experience with completing many research tasks (Finch et al., 2013; Queensland Government, 2013; Stephens et al., 2008). In the present study, reduced confidence manifested as a fear of the unknown (i.e., research), which then impeded research engagement. Indeed, participants in the present study who were not engaged in research often reported a lack of experience and confidence with research tasks and in the absence of support an inability to "get started". This is consistent with literature reporting correlations between positive attitudes towards EBP and self-reported use of EBP, with SLPs who reported less positive attitudes towards EBP less likely to engage in EBP (Zipoli \& Kennedy, 2008).

A lack of research training was viewed as a key obstacle preventing participants who were not engaged in research from partaking in research-related activities. Even those participants who selfreported as engaged in research expressed a desire for further training, particularly in skills such as writing ethics applications and statistics. This observation supports the idea that research skills are a different skill set to clinical skills and that there continues to be new, more advanced research skills to acquire over time. Indeed, this ongoing acquisition of more advanced research skills is reflective of the typical formal, tertiary research pathway of doctoral student, to postdoctoral researcher, to research fellow and then to more senior research roles. The key then may be to translate this practical, experience-based research skill development pathway into a clinical context.

In the present study, participants' individual clinical contexts greatly influenced their level of research engagement. In some cases this influence was positive, with some clinical environments described as providing a rich opportunity for generating research ideas and data collecting; as having line managers who actively supported their staff engaging in research, dedicated positions or portfolios within departments to encourage and facilitate research activities or being active in research training. Negative workplace influences were also discussed, with both engaged and non-engaged participants reporting research constraints stemming from their organisation. Such findings are 
concordant with previous studies of EBP implementation where the workplace environment has been documented as a barrier (O'Connor \& Pettigrew, 2009).

A lack of time for research featured prominently in the present study as a key obstacle preventing research, and was consistent with previous studies which have revealed that a lack of time is the primary barrier to EBP implementation (Jette et al., 2003; Nail-Chiwetalu \& Bernstein Ratner, 2007; O'Connor \& Pettigrew, 2009). Vallino-Napoli and Reilly (2004) revealed that eighty-eight percent of SLPs surveyed believed that SLPs do not have time for research, while a recent government-based survey revealed that allied health professionals spend approximately 0.05 of their full time equivalent position on research during their average week (Queensland Government, 2013). These findings suggest that to increase the research engagement of the SLP workforce, there maybe a need for change at an organisational level to provide dedicated research time for clinicians to pursue research activities. Another potential solution could be for clinicians to share the research workload by having less experienced clinicians assist more senior clinicians with research tasks, thereby not only sharing the workload but also enabling research skills to be acquired by less experienced staff.

Additional obstacles originating from participants' clinical environments in the present study included insufficient availability of research skills (e.g., statistical advice), poor information dissemination about current research projects, specific caseloads perceived as not conducive to research, and poor organisational support for actually implementing research projects. Based on the findings of the current study and previous research (Finch et al., 2013; Stephens et al., 2008), engagement in research by clinical SLPs does not appear to relate exclusively to interest levels. Therefore, engaging clinical SLPs as part of the research workforce is not a matter of merely stimulating interest levels, but also needing to address perceived organisational challenges and skill development. As Reid et al (2006) concluded after finding a similar pattern in primary healthcare workers, a global approach is required to build research capacity in the healthcare setting.

Issues related to research capacity in the current study were divided into two areas: skill development to break down the fear of doing research, and organisational change that would see the 
clinical context shaping research. In the present study, one key strategy being successfully used to break down the fear preventing research engagement was experiential research training. The opportunity to experience research without full responsibility for the project enabled new clinical researchers to become involved, receive support and training, and develop confidence in specific tasks. This suggests that to increase research engagement, we need to provide SLPs with positive, supported research training that enables them to acquire essential research skills and a sense of confidence with undertaking research tasks. Other strategies that have been suggested to increase research capacity include encouraging SLPs to undertake research higher degrees (Finch et al., 2013; Iles \& Davidson, 2006; MacEwan Dysart \& Tomlin, 2002), establishing links with universities (Reid et al., 2006), creating allied health dedicated research positions in healthcare settings, and mentoring with more experienced colleagues (Queensland Government, 2013: Reid et al., 2006). Additional support could be achieved by establishing a local or national research special interest group for SLPs engaged in research activities to share their experiences with likeminded colleagues (Reid et al., 2006). Interestingly, many participants in the present study, both engaged and not engaged in research, were interested in participating in a research special interest group.

The present study illustrated that the timing of experiential research training and special interest groups is vital. It is imperative that the timing for accessing support and skills training is carefully considered to avoid a disconnection between the acquisition of knowledge and the actual implementation of this knowledge and skill. Many participants in the present study reported that some research skills training was received during their undergraduate university training; however, retention and translation of this knowledge into research activity was a challenge. In the allied health field of EBP skills, Iles and Davidson (2006) found that recent physiotherapy graduates reported higher confidence in their EBP skills as graduates compared to those who had graduated several years previously. This highlights the need for practical, experiential research training at a time when the specific research skills are actually required.

One pivotal thread woven through the present study was the influence of personal factors. In the present study, participants engaged in research typically reported overcoming a lack of knowledge 
and experience with more complex research tasks by actively seeking out collaborations, mentoring and training in specific skills. Personal factors also determined how participants responded to the opportunities and challenges presented by their clinical context. Again, participants engaged in research tended to proactively seek out solutions to these challenges through avenues such as collaborating with colleagues and university staff. However that said, not all participants engaged in research were required to actively seek out solutions to challenges; instead some participants were simply taking advantage of opportunities offered by their clinical environment (such as data collection that involved their routine clinical activities). These observations are consistent with previous research which has found correlations between interest and research engagement (Stephens et al., 2008), and between attitudes towards EBP and actual EBP engagement (Zipoli \& Kennedy, 2008). This suggests that individuals who are proactive, have positive attitudes towards research and are interested in research may be more likely to engage in research activities. Additionally, it suggests that any strategy which is introduced to increase research capacity must consider the individual SLP's interest, confidence with research and level of self-direction, when determining the nature and amount of support provided.

\section{Limitations}

The present study was limited by the restriction of participants to a single employing organisation. That said; however, the organisation targeted in this study is the largest employer of SLPs in the state of Queensland, Australia. Although turn taking in each focus group was actively balanced by the group moderator, the mixture of teleconferencing and videoconferencing (with some face-to-face participants) may have also influenced the study results. However, given that the research team was interested in capturing the experiences and perspective of SLPs from a variety of workplaces throughout Queensland and the vast geographical size of the state, it was not possible for all participants to be physically present in the same room for the focus groups. The small numbers of participants in the focus groups may have limited the study results; however, data collection continued 
until saturation occurred and no new information emerged. We acknowledge that there were more research engaged than non-research engaged participants in the study; however, we employed purposive sampling for recruitment so our aim was to recruit participants who were and were not engaged in research. Specifically, we were interested in capturing a variety of experiences and perceptions towards conducting clinical research, rather than specific numbers of participants in each group. Finally, while designed to enable each viewpoint to be explored comprehensively without being overshadowed, the division of the focus groups into research engaged participants and nonresearch engaged participants may have influenced the flow of the discussion. It is possible that different discussions may have occurred had the groups been combined.

\section{Conclusion}

The present study revealed that engagement in research is shaped by a range of factors, although key drivers include whether clinicians experience a fear of research and their organisational context. Mediating these factors was the influence of individual personal factors, such as proactivity and initiation. Providing opportunities for SLPs to participate in supported research training that takes into account the individual characteristics of each clinician may help to increase the research engagement of the SLP workforce. Manipulating the clinical context to ensure it encourages and supports research may further increase research engagement. This in turn may assist healthcare providers to develop an allied health workforce that not only adheres to EBP but also actively generates new evidence to guide the future of the workforce. As one participant stated: "It's not only the formal research we engage in that we publish in the end, but...having an inquisitive mind about our process and wanting to improve it is vital... To get that culture started with a new batch of clinicians is probably the best thing that you can do."

\section{Acknowledgements}


The authors would like to thank all of the participants who gave up their valuable time to share their experiences with us.

\section{Declaration of interest}

The authors report no conflicts of interest. The authors alone are responsible for the content and writing of the paper.

\section{References}

Australian Government. (2013). Strategic review of health and medical research - Better health through research. Australian Government: Department of Health and Ageing.

Australian Physiotherapy Association. (n.d.a.) Research. Retrieved December 9, 2013, from http://www.physiotherapy.asn.au/APAWCM/Research_and_Publications/APAWCM/Research_and_ Publications/APA_Portal.aspx?hkey=07b5740c-71c9-4148-8d0f-024a862c384e

Canadian Institutes of Health Research (2004). Innovation in Action: Knowledge translation strategy 2004 - 2009. Retrieved February 19, 2014 from www.cihr-irsc.gc.ca/e/44570.html

Coenen, M., Stamm, T. A., Stucki, G., \& Cieza, A. (2012). Individual interviews and focus groups in patients with rheumatoid arthritis: a comparison of two qualitative methods. Quality of Life Research, 21, $359-370$.

Finch, E., Cornwell, P., Ward, E., \& McPhail, S. (2013). Factors influencing research engagement: research interest, confidence and experience in an Australian speech-language pathology workforce. BMC Health Services Research, 13:144, http://www.biomedcentral.com/1472- 
Graneheim, U. H., \& Lundham, B. (2004). Qualitative content analysis in nursing research: concepts, procedures and measures to achieve trustworthiness. Nurse Education Today, 24, 105-112.

Iles, R., \& Davidson, M. (2006). Evidence based practice: A survey of physiotherapists' current practice. Physiotherapy Research International, 11(2), 93-103.

Jette, D. U., Bacon, K., Batty, C., Carlson, M., Ferland, A., Hemingway, R. D., Hill, J. C., Ogilvie, L., \& Volk, D. (2003). Evidence-based practice: Beliefs, attitudes, knowledge, and behaviors of physical therapists. Physical Therapy, 83(9), 786-805.

Kitson, A., Powell, K., Hoon, E., Newbury, J., Wilson, A., \& Beilby, J. (2013). Knowledge translation within a population health study: how do you do it? Implementation Science, 8:54. http://www.implementationscience.com/content/8/1/54

Koch, T. (2006). Establishing rigour in qualitative research: the decision trail. Journal of Advanced Nursing, 53(1), 91-100.

Krueger \& Casey (2000). Focus groups: A practical guide for applied research $\left(3^{\text {rd }}\right.$ ed). Thousand Oaks: Sage.

Marshall, M. N. (1996) Sampling for qualitative research. Family Practice, 13(6), 522-525.

MacEwan Dysart, A. M., \& Tomlin, G. S. (2002). Factors related to evidence-based practice among U.S. occupational therapy clinicians. American Journal of Occupational Therapy, 56, 275284.

Nail-Chiwetalu, B., \& Ratner, N. B. (2007). An assessment of the information-seeking abilities and needs of practicing speech-language pathologists. Journal of the Medical Library Association, 95(2), 182-E57.

NHMRC. (2013, January 10) Research translation. Retrieved July 26, 2013, from http://www.nhmrc.gov.au/research-translation 
O'Connor, S. \& Pettigrew, C., M. (2009). The barriers perceived to prevent the successful implementation of evidence-based practice by speech and language therapists. International Journal of Language and Communication Disorders, 44(6), 1018-1035.

Occupational therapy Australia. (2013). Vision and purpose. Retrieved December 9, 2013, from http://www.otaus.com.au/about/vision-purpose

Peters, D. H., Tran, N. T., \& Adam, T. (2013). Implementation research in health: a practical guide. Alliance for Health Policy and Systems Research, World Health Organization: Geneva, $\underline{\text { Switzerland. }}$

Queensland Government (2012). Centre for Functioning and Health Research. Last reviewed 13 August 2012. www.health.qld.gov.au/cfahr/ Accessed 28 August 2013.

Queensland Government (2013). Allied health professions office of Queensland - Health practitioner research capacity survey 2012. Version 1.0. July 2013. Brisbane, Qld: Queensland Government.

Queensland Industrial Relations Commission (2007). Health practitioners (Queensland Health) certified agreement (No. 1) 2007. Retrieved 15 November 2011 from www.health.qld.gov.au/eb/current_agree.asp.

Reid, K., Farmer, E. A., \& Weston, K, A. (2006). Setting directions for capacity building in primary health care: A survey of a research network. BioMed Central Family Practice, 7(8), http://www.biomedcentral.com/1471-2296/7/8

Reid, K., Farmer, E. A., \& Weston, K, A. (2007). Bursaries, writing grants and fellowships: A strategy to develop research capacity in primary health care. BioMed Central Family Practice, 8(19), http://www.biomedcentral.com/1471-2296/8/19

Speech Pathology Australia (2011). Competency-Based Occupational Standards (Entry Level). Speech Pathology Australia: Melbourne, Victoria. 
Speech Pathology Australia. (2012). Speech Pathology Australia. Retrieved December, 9, 2013, from www.speechpathologyaustralia.org.au

Stephens, D., Taylor, N., \& Leggat, S. G. (2008). Research experience and research interests of allied health professionals. Journal of Allied Health, 38(4), e107-e111.

Straus, S. E., Tetroe, J., \& Graham, I. (2009). Defining knowledge translation. Canadian medical Association Journal, 181 (3 -4), 165 - 168.

Vallino-Napoli, L. D., \& Reilly, S. (2004). Evidence-based healthcare: A survey of speech pathology practice. Advances in Speech-Language Pathology, 6(2), 107-112.

Zipoli, R. P., \& Kennedy, M. (2005). Evidence-based practice among speech-language pathologists: Attitudes, utilization, and barriers. American Journal of Speech-language Pathology, 14, 208-220. 